\title{
Getter sputtering system for high- throughput fabrication of composition spreads
}

Cite as: Rev. Sci. Instrum. 78, 072212 (2007); https://doi.org/10.1063/1.2755967

Submitted: 03 November 2006 . Accepted: 13 February 2007 . Published Online: 13 July 2007

John M. Gregoire, R. B. van Dover, Jing Jin, Francis J. DiSalvo, and Héctor D. Abruña

ARTICLES YOU MAY BE INTERESTED IN

High throughput screening of electrocatalysts for fuel cell applications

Review of Scientific Instruments 77, 054104 (2006); https://doi.org/10.1063/1.2202919

Applications of high throughput (combinatorial) methodologies to electronic, magnetic, optical, and energy-related materials

Journal of Applied Physics 113, 231101 (2013); https://doi.org/10.1063/1.4803530

Plasma sputtering system for deposition of thin film combinatorial libraries

Review of Scientific Instruments 76, 062221 (2005); https://doi.org/10.1063/1.1921552

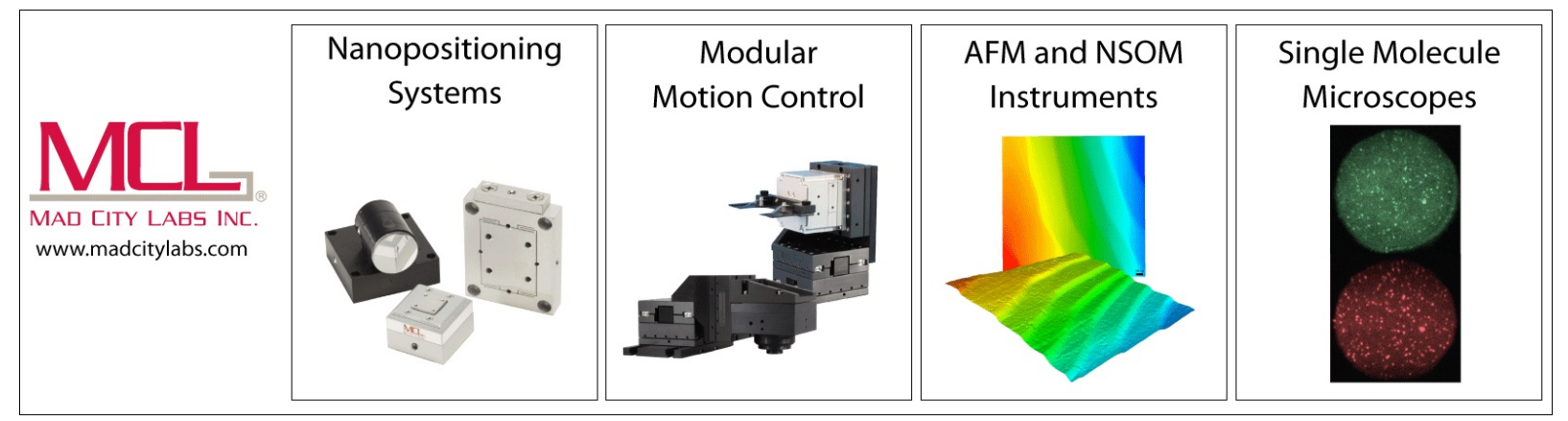

Rev. Sci. Instrum. 78, 072212 (2007); https://doi.org/10.1063/1.2755967

78, 072212

(c) 2007 American Institute of Physics. 


\title{
Getter sputtering system for high-throughput fabrication of composition spreads
}

\author{
John M. Gregoire \\ Department of Physics, Cornell University, Ithaca, New York 14853 \\ and Cornell Fuel Cell Institute, Cornell University, Ithaca, New York 14853 \\ R. B. van Dover \\ Department of Materials Science and Engineering, Cornell University, Ithaca, New York 14853 \\ and Cornell Fuel Cell Institute, Cornell University, Ithaca, New York 14853 \\ Jing Jin, Francis J. DiSalvo, and Héctor D. Abruña \\ Department of Chemistry and Chemical Biology, Cornell University, Ithaca, New York 14853 \\ and Cornell Fuel Cell Institute, Cornell University, Ithaca, New York 14853
}

(Received 3 November 2006; accepted 13 February 2007; published online 13 July 2007)

\begin{abstract}
We describe a sputtering system that can deposit composition spreads in an effectively UHV environment but which does not require the high-throughput paradigm to be compromised by a long pump down each time a target is changed. The system deploys four magnetron sputter guns in a cryoshroud (getter sputtering) which allows elements such as $\mathrm{Ti}$ and $\mathrm{Zr}$ to be deposited with minimal contamination by oxygen or other reactive background gases. The system also relies on custom substrate heaters to give rapid heating and cool down. The effectiveness of the gettering technique is evaluated, and example results obtained for catalytic activity of a pseudoternary composition spread are presented. (C) 2007 American Institute of Physics. [DOI: 10.1063/1.2755967]
\end{abstract}

\section{INTRODUCTION}

The fabrication of combinatorial libraries of inorganic materials is an increasingly popular technique employed in materials optimization and discovery. The study of continuous composition spreads was developed by Kennedy et al. ${ }^{1}$ in 1965 and, in the context of cosputtering (simultaneous deposition from multiple sputter sources), by Sawatzky and $\mathrm{Kay}^{2}$ in 1969. In the past few years, several new deposition techniques, such as the printing of salts for discrete composition spreads ${ }^{3}$ and the controlled diffusion of gels for continuous composition spreads ${ }^{4,5}$ have been introduced. However, the thin film composition spread has remained an important technique, and the present work contributes to its active development.

The primary inhibition to the widespread use of composition spreads in the decades following their introduction was the lack of sufficiently automated measurement tools for evaluation of the materials. The introduction of computerassisted evaluation in the 1990s marked an important increase in the use of combinatorial methods. ${ }^{6-8}$ Among these efforts, Chang et al. ${ }^{9}$ developed an alternative to cosputter deposition. Their technique utilizes shutter and/or substrate movement to create a film with a thickness gradient, i.e., a wedge. Sequential deposition of overlapping wedges from any number of sources yields a film that, upon annealing, can form a continuous composition spread. The main advantage of this technique is control over the shape and steepness of the composition gradients, as these film properties are determined by the computer-controlled shutter and substrate motion. However, while the thickness of each wedge can be made arbitrarily small in principle, the deposited material lacks the intimate, atomic-scale mixing that codeposition provides. While annealing may address this issue, the need to anneal greatly limits the ability to independently control the film microstructure and crystal chemistry.

An important concern in any deposition technique is the purity of the fabricated material. In physical vapor deposition, the most common cause of film contamination is the incorporation of reactive gases in the film. While performing depositions in an ultrahigh vacuum (UHV) virtually eliminates this concern, the common UHV practices of using load locks and performing long pump downs after system venting are inhibitive for many studies. In particular, for combinatorial, high-throughput studies, the need to frequently change substrates and targets makes UHV highly impractical. In 1964, Theuerer and Hauser ${ }^{10}$ introduced a technique for reducing the reactive gas partial pressure in their vacuum chamber capable of reaching a base pressure no lower than $10^{-6}$ Torr. They enclosed their single sputter source and substrate in a metal can, which during deposition was cooled with liquid nitrogen. Any reactive gases within the enclosure then reacted with the material deposited on the enclosure walls and remained there due to the high sticking coefficient created by the cryogenic temperature of the metal. In 1985, van Dover et al. ${ }^{11}$ extended this so-called getter sputtering technique to a three-source deposition system for studying linear composition spreads of amorphous transition metal-rare earth alloys. To the authors' best knowledge, the current work is the first to employ the gettering technique in a combinatorial system capable of deposition of ternary composition spreads. In addition, this work incorporates the gettering technique in a system designed for high-throughput synthesis. 


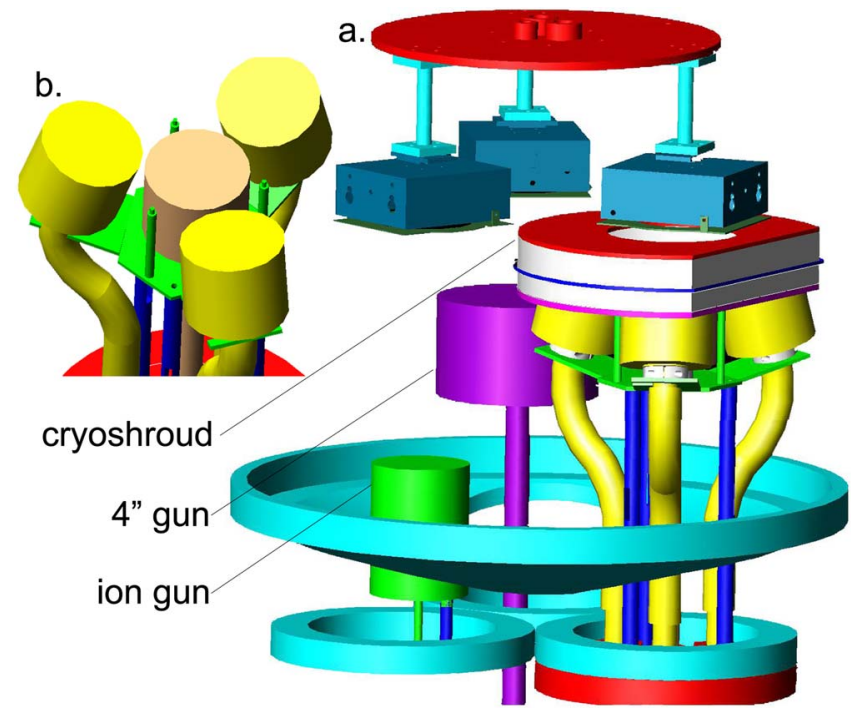

FIG. 1. (Color online) (a) The three custom substrate heaters on their rotating mounting plate are pictured with the ion gun, 4 in. gun, and four-gun assembly as positioned in the deposition system. (b) A detailed look at the arrangement of the four-gun assembly.

In the context of fuel cell anode catalyst materials, the ability to control the concentration of reactive gases in a sputtered material is important, for even modest concentrations will affect film microstructure and may inhibit the growth of ordered intermetallic phases. Casado-Rivera et al. ${ }^{12,13}$ demonstrated that attention to such details is prudent through their discovery of binary ordered metallic phases that show not only high activity for the electrooxidation of formic acid but also resilience to poisons commonly found in fuel cell operating environments. It is also worth noting that substrate temperature during deposition is a key parameter in controlling film chemistry and microstructure and thus special attention must be given to substrate heating.

Prochaska et al. ${ }^{14}$ introduced high-throughput, thin film combinatorial synthesis and evaluation methods to the search for electroactive intermetallics and alloys containing two or three elements. The instrument described in the current work contributes to the search for superior catalyst materials by providing additional thin film fabrication abilities, due most notably to its cryoshroud and custom-made radiative heaters. Also, the system is designed to provide an order of magnitude increase in the rate of composition spread production. In this article, the main features of the deposition system and deposition techniques are described, followed by experimental results that demonstrate the effectiveness of novel techniques.

\section{SYSTEM DESIGN}

\section{A. Combinatorial film synthesis}

The sputter deposition system contains four 2 in. magnetron sputter guns (Angstrom Sciences Onyx-2), a 4 in. AJA, Inc. magnetron sputter gun, and a $2.5 \mathrm{~cm}$ IonTech, Inc. ion gun, all pictured in Fig. 1(a) as mounted in the vacuum chamber. Also seen in Fig. 1(a) is a round plate that holds three substrate heaters for 3 in. substrates. The plate is at- tached to a rotary feedthrough that is geared to a stepper motor. This setup allows for computerized movement that brings any of the three substrates to any of the three film processing positions described in the following paragraphs.

The 4 in. gun is used for making highly uniform films. Most commonly, it is used for depositing a tantalum adhesion layer on silicon substrates prior to the deposition of a composition spread. Such uniform metallic layers are also important in substrate heating, as will be discussed in Sec. II B. A stainless steel chimney (not shown) is mounted atop this gun to keep the sputtered material from being deposited anywhere other than on the substrate and its holder.

The ion gun is used for substrate cleaning, for example, to remove the insulating $\mathrm{SiO}_{2}$ layer from silicon wafers. In conjunction with the substrate motion, it may also be useful for controlling film microstructure by employing deposition techniques involving alternations between sputtering and ion milling.

The four-gun assembly [Fig. 1(b)] is used for deposition of thin film composition spreads via cosputtering. One of the 2 in. guns is centered below the substrate position in a standard on-axis orientation. The remaining three 2 in. guns are positioned around the central gun, each with a confocal $15^{\circ}$ tilt. In addition to the common binary and ternary composition spreads, this ensemble allows for codeposition of pseudoternary composition spreads: composition spreads containing four elements and covering a region in composition space with the dimensionality of a ternary spread.

The tilt of the three outer guns provides smooth composition gradients as a function of lateral distance on the substrate surface. Without the $15^{\circ}$ tilt, sputtered atoms leaving the target would reach the most distant region of the substrate primarily via scattering events with the ambient gas. The resulting angle of incidence at the substrate can be quite sharp for such sputtered material, and unexpected and largely uncontrollable microstructure may ensue. This particular arrangement of the guns was chosen to minimize such effects while also keeping the material sputtered in a direction normal to the plane of the target from being deposited on the substrate. This latter geometrical provision is important for the existence of composition gradients across the entire substrate and is also important in the case of deposition in an oxygen environment, when sputtering of the substrate by $\mathrm{O}^{-}$ commonly occurs.

Between each pair of substrate heaters is a circular aluminum sheet (not pictured) that can be rotated over the 4 in. gun or four-gun assembly for presputtering. At the center of one of the sheets is mounted an Inficon crystal deposition monitor that measures the mass accumulation rate at a given point with better than $10 \mathrm{ng} / \mathrm{cm}^{2}$ resolution. A direct measure of the profile of deposition rate for each gun can be obtained by incrementally rotating the deposition monitor along an arc above the appropriate gun. For the gun nearest to the center of the chamber, this technique does not provide much information about the deposition profile because the rate remains nearly constant throughout the crystal monitor's path. However, for each of the remaining three guns in the four-gun ensemble, deposition rates as a function of crystal monitor position are used to fit an empirical form for the 
deposition profile perceived by a 3 in. substrate. Since each of the three confocally tilted guns yields an identical deposition profile for a given target with a given applied power, this technique allows the user to quickly calculate the expected composition of an entire composition spread. This method for determining position-dependent film composition is usually accurate to within several at. \%, rivaling profilometry-based techniques and standardless energy dispersive x-ray analysis. Codeposition of certain sets of elements can yield compositions that differ significantly from those predicted by this method if any of the sputtered elements have a high vapor pressure at the substrate temperature or if the sticking coefficient of any of the elements is compromised by resputtering from the substrate by one of the other elements or by matrix effects in the film. The former of these two conditions cannot be explored with the deposition monitor because the monitor's temperature cannot be systematically controlled. The deviations from calculated compositions can, however, be easily anticipated by reviewing the published vapor pressure data. The latter condition will have some dependence on substrate temperature but can be explored at ambient temperature by using the deposition monitor to measure the extent by which the mass accumulation rate achieved by cosputtering a set of elements differs from the sum of the individual rates.

The final item of note in Fig. 1 is the stainless steel cryoshroud that encloses the region between the deposition sources and the substrate. The walls have four openings on the bottom which follow the contours of the three tilted and one central gun and one centered hole on top which allows sputtered material to reach the substrate. It is worth noting that the top hole must be sufficiently large to avoid any shadowing problems. A single loop of 0.25 in. stainless steel tubing is welded to the cryoshroud walls and attaches to flexible stainless steel bellows that lead to thermally isolated feedthroughs. While the original getter ${ }^{10}$ formed a tight seal except for one argon inlet point, the getter in Fig. 1 is only loosely sealed with the sputter guns and substrate holder. It simply rests on posts welded to the same plate assembly that holds the four guns. This arrangement in combination with the flexible bellows allows for quick and easy removal of the cryoshroud during target changes. In fact, the design of this system allows target changes to be performed in less than 5 min.

To start a typical deposition process, three 3 in. silicon wafers are mounted in the substrate holders and the system is pumped to the low $10^{-7}$ Torr range with a CryoTorr8 vacuum pump. The substrates are sequentially brought to the desired deposition temperatures while the tantalum adhesion layer is deposited. Simultaneously, the cryoshroud is cooled with flowing liquid nitrogen. Typical cryoshroud cool down times are $10-20$ min depending on the nitrogen flow rate. Once tantalum deposition is complete, the composition spread is deposited. All depositions occur in $5 \mathrm{mT}$ argon, regulated with a feedback-controlled angle valve in the high vacuum pumping line. The deposition monitor is commonly used before and after each deposition to ensure that the deposition sources are performing as expected.
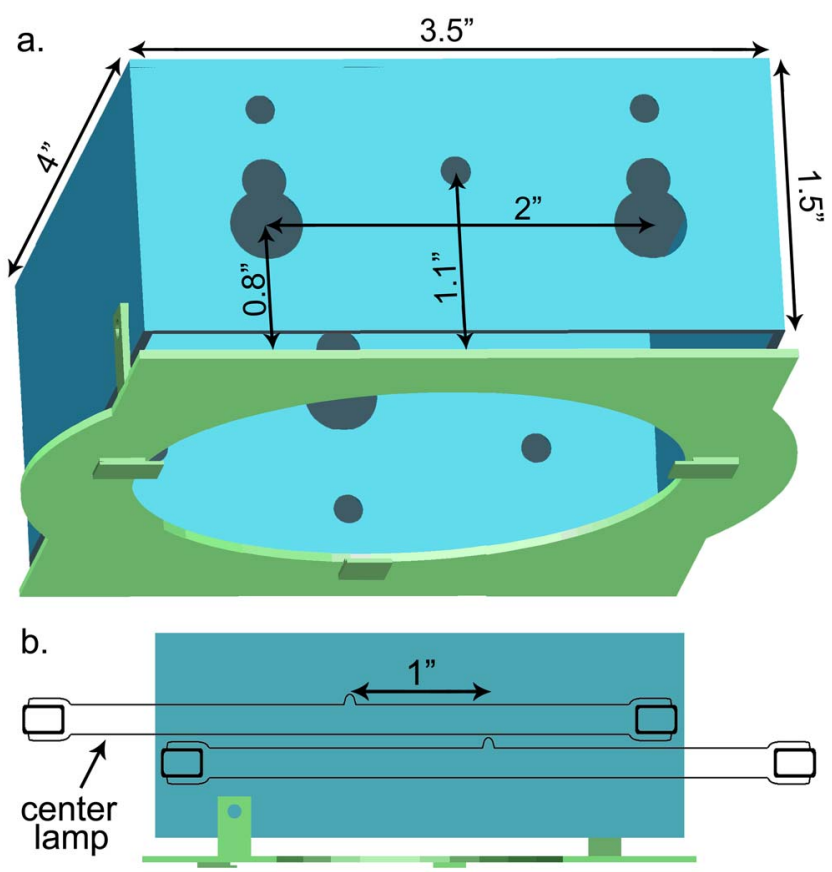

FIG. 2. (Color online) (a) The custom heater box is shown with the substrate holder plate. Dimensions for the heater box and for the positions of the three lamps with respect to the plane of the substrate are given. The lamps are inserted into the larger ("8"-shaped) holes. (b) This side view of the heater shows the position of the center lamp with respect to the outer lamps. Inserting the center lamp in the opposite side from the two outer lamps provides a 1 in. separation between the center of the inner and outer lamps.

\section{B. Custom substrate heaters}

The three substrate heaters were designed and built inhouse. The heater design focuses on providing uniform substrate temperature, rapid warming and cooling cycles, and high heating efficiency, i.e., minimum power required to achieve and maintain a given substrate temperature. These three criteria are superbly met with a design that utilizes three $500 \mathrm{~W}$ halogen quartz lamps. The use of domestic lamps offers low cost, quick and highly reproducible filament changes, and an enclosed tungsten filament that allows for operation in an oxygen environment.

While single-lamp units for in-vacuum substrate heating are commercially available, no practical installation of such a unit yields uniform substrate heating. Thermodynamic simulations were performed to determine the three-lamp configuration that best meets the need for uniform heating across an entire 3 in. wafer while maintaining high heating efficiency.

\section{Heater design}

The heater box and substrate holder, shown in Fig. 2, are formed from sheets of $0.04 \mathrm{in}$. stainless steel. Sheets of 0.002 in. molybdenum line the interior of the heater box, serving as reflectors. The heater box holds three lamps in the appropriate positions with spring-loaded electrical connections to avoid contact loss due to differential thermal expansion.

The substrate holder plate is attached to the heater box with two small tabs, minimizing thermal contact. With heaters positioned as depicted in Fig. 1(a), the three tabs on the substrate plate are sufficient for holding the substrate. This 


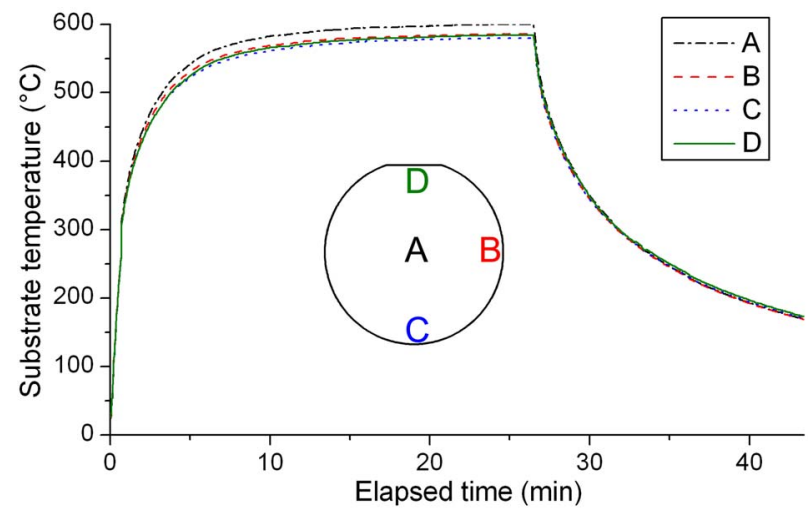

FIG. 3. (Color online) The inset shows four points at which thermocouples are cemented to a 3 in. silicon wafer. The temperatures obtained from the four thermocouples are plotted as the substrate is heated by a custom heater under $100 \mathrm{~W}$ applied power. The beginning of the cooling profile is shown after $26 \mathrm{~min}$ of heating.

method of substrate mounting and the low thermal mass of the entire heater assembly allow for rapid substrate cooling. While the basic design parameters are given in Fig. 2, drawings, photographs, and further description are available online. $^{15}$

\section{Heater performance}

Figure 3 demonstrates the typical uniformity in substrate heating; maximum temperature variations on a 3 in. wafer are limited to a few percent of the deviation from room temperature up to the maximum tested temperature of $600{ }^{\circ} \mathrm{C}$.

A common problem with in-vacuum heating is that heat dumped into the system causes increased outgassing, which raises the partial pressure of reactive gases. While the situation is improved by performing a heating cycle during pump down, this time-intensive procedure fails to fully solve the problem. Thus, for a given desired substrate temperature, important heater specifications are the total electrical energy required to bring the substrate to the elevated temperature and the power required to maintain the elevated temperature. For a substrate temperature of $400{ }^{\circ} \mathrm{C}$, our custom heaters require $27 \mathrm{~kJ}$ for a $5 \mathrm{~min}$ warm up, compared to the hundreds of kilojoules required for commercially available Ohmic heaters, which additionally can require warm up times in excess of $30 \mathrm{~min}$. The heaters can maintain the $400{ }^{\circ} \mathrm{C}$ temperature with only $65 \mathrm{~W}$ applied power.

An additional attribute of this heater design that makes it particularly appealing to high-throughput studies is exceedingly short cool-down times. The low thermal mass of the heater and the minimal thermal contact to the substrate allow for substrate cooling from 400 to below $100{ }^{\circ} \mathrm{C}$ in $30 \mathrm{~min}$. The quick decline from high temperatures also shortens the time in which a sputtered film may react strongly with reactive gases in the chamber. The remaining notable feature is a control circuit containing a thermocouple, proportionalintegral-derivative (PID) controller, relay, and transformer, which provides automated temperature control to a userspecified setpoint.

It is worth noting that the data on heater performance were obtained using silicon wafers coated with tantalum. Bare silicon and other lightly absorbing media are not heated as efficiently. When deposition on such substrates is required, the back of the substrate must first be coated with an absorbing material.

\section{RESULTS AND DISCUSSION}

\section{A. Film purity}

Quantifying the effectiveness of the gettering technique in removing reactive gases from the sputtering environment is difficult. Commonly, the reactive gases of concern are hydrocarbons, water vapor, and diatomic oxygen, and therefore one would like to measure the partial pressure of these gases within the cryoshroud during sputtering while the absolute pressure is 5 mTorr. To gather information on these quantities, we have performed two studies using deposition of the oxyphyllic element titanium. The studies are meant to provide information on $P_{\mathrm{O}}$, the effective partial pressure of all oxygen-containing gases.

\section{WDS analysis}

The first study compared oxygen incorporation in titanium films with and without the cooled cryoshroud. Film deposition commenced 35 min after the start of rough pumping, which provided a chamber pressure of $3 \mu$ Torr in the no-gettering case. During this time, the $\mathrm{SiO}_{2}$ was ion etched from the silicon substrate, the substrate was brought to the $400{ }^{\circ} \mathrm{C}$ deposition temperature, and, where applicable, the cryoshroud was cooled for $10 \mathrm{~min}$. Titanium was deposited from one of the angled 2 in. guns onto the silicon substrate creating a film with center thickness of $40 \mathrm{~nm}$. The film was then capped with $15 \mathrm{~nm}$ of Pt deposited from the centered 2 in. gun to minimize film oxidation upon exposure to air. The film was allowed to cool in vacuum and was analyzed using standard-calibrated wavelength dispersive spectroscopy (WDS). The program GMRFILM ${ }^{16}$ was used for electron Monte Carlo and x-ray absorption adjustments to the WDS raw data, providing the calculated at. \% oxygen present in the films. Figure 4 shows the WDS results as a function of deposition rate. In the limit that the sticking coefficient of oxygen is independent of $P_{\mathrm{O}}$, the atomic percentage of oxygen present in a sputtered film should be proportional to $P_{\mathrm{O}} / r$, where $r$ is the deposition rate. The least-squares fit for this functional form is given for each set of data in Fig. 4. The ratio of the fit parameters suggests that the use of the cryoshroud reduced the effective $P_{\mathrm{O}}$ by a factor of 4.5. This type of analysis was not performed at lower absolute pressures (longer pump down times) because the signal to noise ratio for the WDS analysis becomes very poor with lower oxygen incorporation.

\section{Film resistivity analysis}

To test the effectiveness of the gettering technique for films sputtered in a fully pumped system (absolute base pressure of approximately $0.3 \mu$ Torr), resistivity analysis was performed. The film resistance of a metallic titanium film of given thickness is caused by electronic scattering primarily with phonons, impurity atoms, and titanium grain boundaries. The first of these has a strong temperature dependence, while the latter two are independent of temperature. Thus, 


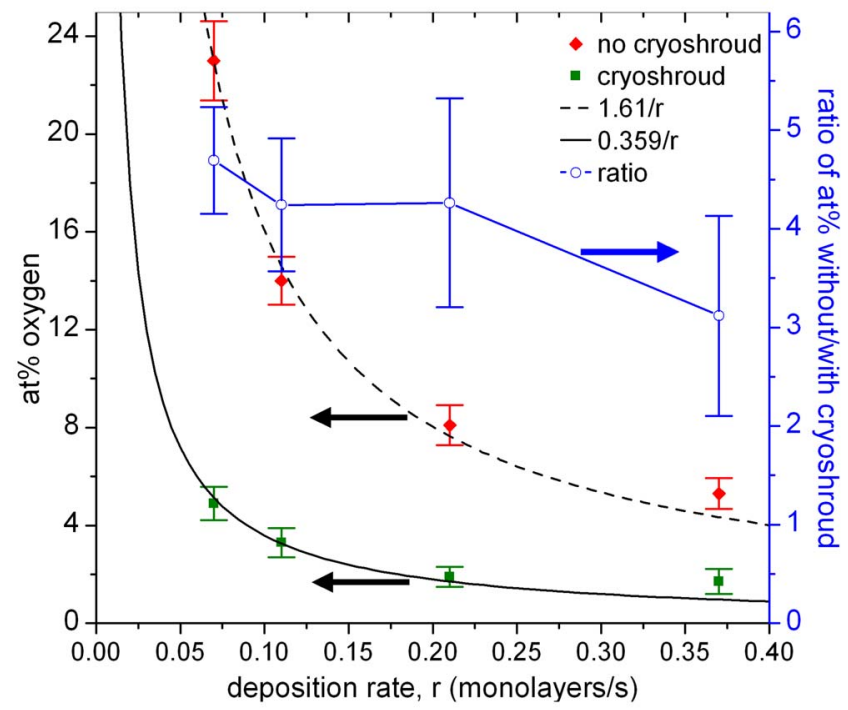

FIG. 4. (Color online) The at. \% oxygen in sputtered titanium films, as determined by WDS measurements, is plotted for depositions both with and without the cryoshroud. The orientation of the gun allows for analysis of a range of deposition rates with a single deposition. Rates are quoted as monolayers of close packed titanium per second. Fitted functions and the ratio of at. \% oxygen without the cryoshroud to that with the cryoshroud are also plotted.

the ratio of the film resistivity at $300 \mathrm{~K}$ to that at $5 \mathrm{~K}$ can be used as a measure of film quality. This resistance ratio has a minimum of unity for films whose resistances are dominated by contaminant atom and grain boundary scattering.

Titanium films were once again deposited at $400{ }^{\circ} \mathrm{C}$ both with and without the cryoshroud. For each film, the deposition rate was fixed at $\sim 0.5 \mathrm{~nm} / \mathrm{s}$. In the gettering deposition, the chamber base pressure during sputtering remained in the mid- $10^{-7}$-Torr range, while this pressure rose to the low $10^{-6}$ Torr range during the deposition without the cryoshroud. Standard four-point van der Pauw techniques ${ }^{17}$ were used to measure film resistivity of $5 \mathrm{~mm}$ square substrate pieces. The resistivity of the film deposited with gettering has a resistance ratio of $4.7 \pm 0.1$, while the film deposited without gettering has a resistance ratio of $3.4 \pm 0.1$. A film deposited with the same parameters in a deposition system using Ohmic heaters which force the chamber base pressure during sputtering to nearly near $10^{-5}$ Torr has a resistance ratio of $1.2 \pm 0.1$. We thus conclude that our custom heaters, and to even greater extent the gettering technique, improve the quality of the titanium films deposited in a highthroughput (non-UHV) setting. Quantifying oxygen contamination from the resistance ratio measurements would require numerically distinguishing resistance due to electroncontaminant atom scattering from that due to electron-grain boundary scattering, which we do not attempt here.

\section{B. Example electrochemical results from a pseudoternary composition spread}

To illustrate other typical film properties, we present the preliminary results of a single pseudoternary composition spread tested for catalytic activity for the oxidation of methanol. The testing method used is the high-throughput fluorescence test described in detail previously ${ }^{14}$ which yields im- a.
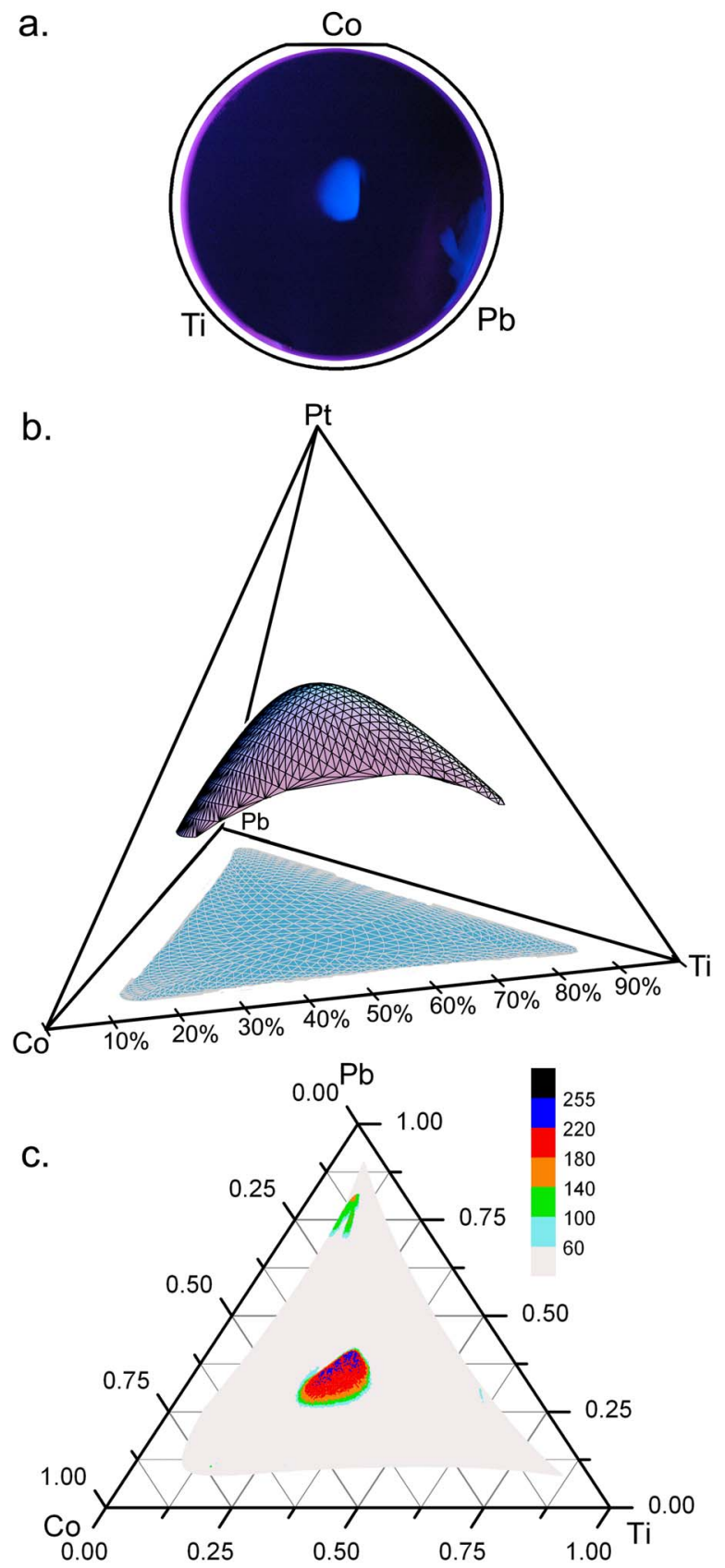

FIG. 5. (Color online) (a) A fluorescence image taken during electrochemical testing of the film is shown in the outline of the substrate. The image was taken with a potential of $350 \mathrm{mV} v \mathrm{Ag} / \mathrm{AgCl}$ applied to the film, revealing catalytically active regions in the center and low right. The orientation of the three confocally tilted guns is portrayed by the symbols of the target elements. (b) The region of composition space covered by the film is the dome-shaped surface in $\mathrm{Pt}-\mathrm{Pb}$-Ti-Co composition space. The projection of this surface onto the Pb-Ti-Co composition space is also shown. (c) The intensity of the fluorescence image is mapped onto a ternary composition plot, showing the optimal $\mathrm{Pb}$ :Ti:Co atomic ratios.

ages with catalytically active regions marked by their fluorescence. A composition spread was prepared with $250{ }^{\circ} \mathrm{C}$ substrate temperature with $\mathrm{Pb}, \mathrm{Co}$, and $\mathrm{Ti}$ in the angled guns and $\mathrm{Pt}$ in the centered gun. Deposition rates were chosen such that the center composition would be 50 at. \% Pt and equal atomic ratios among the other three elements. The film composition was calculated using deposition rate techniques described above and the result is given 
in Fig. 5(b). For ease in determining the optimal $\mathrm{Pb}: \mathrm{Ti}: \mathrm{Co}$ ratios, the fluorescence image result of the electrochemical test [Fig. 5(a)] is mapped onto a ternary diagram [Fig. 5(c)]. The center and low-right active regions of Fig. 5(a) have atomic ratios (Pt:Pb:Ti:Co) of approximately $3: 1: 1: 1$ and 4:8:0.8:1, respectively. The applied voltage at which fluorescence was first observed indicates that both of these regions have an onset potential for the oxidation of methanol that is approximately $80 \mathrm{mV}$ more positive than $\mathrm{Pt}$ nanoparticles. The fabrication and testing of this film, which required only a few hours from start to finish, reveal the optimal compositions within the tested region of composition space indicated in Fig. 5(b). While this single experiment did not reveal a breakthrough catalyst, its results direct future investigation of this set of four elements to more Pt-rich composition spreads and to binary and ternary subsets.

\section{Pertinence in combinatorial film deposition}

We present a deposition system designed for cosputtering composition spread libraries. In addition to exploring ternary composition space continuously, the system includes features for controlling film properties. The primary design focus of this new system is to address the issue of reactive gas contamination in deposited films. While the inclusion of a reactive gas in a material may improve material performance, control over inclusion is necessary to conduct a systematic study. To this end, the composition spread is sputtered in a liquid nitrogen cooled cryoshroud which allows for combinatorial, high-throughput studies of materials that may be poisoned by residual gases at microTorr partial pressures. Such materials include those currently used in the study of shape memory alloys, magnetorestrictive materials, semiconductors, and essentially any nonoxide material that includes an oxyphyllic element.

\section{ACKNOWLEDGMENTS}

The authors wish to thank Mark Prochaska, Maxim Kostylev, Sara Barron, Sharon Gerbode, Paul Bishop, and John Sinnot for helpful conversations and general assistance in the construction of the deposition system. The WDS analysis was made possible by the Cornell Center for Materials Research Facilities supported by the National Science Foundation under Award No. DMR-0520404. Primary funding for this work is provided by the Department of Energy, Grant No. DE-FG02-03ER46072.

${ }^{1}$ K. Kennedy, T. Stefansky, G. Davy, V. F. Zackay, and E. R. Parker, J. Appl. Phys. 36, 3808 (1965).

${ }^{2}$ E. Sawatzky and E. Kay, IBM J. Res. Dev. 13, 696 (1969).

${ }^{3}$ M. M. Mohebi and J. R. G. Evans, J. Comb. Chem. 4, 267 (2002).

${ }^{4}$ S. Jayaraman and A. C. Hillier, J. Comb. Chem. 6, 27 (2004).

${ }^{5}$ S. Jayaraman and A. C. Hillier, Meas. Sci. Technol. 16, 5 (2005).

${ }^{6}$ X.-D. Xiang et al., Science 268, 1738 (1995).

${ }^{7}$ R. B. van Dover, L. F. Schneemeyer, and R. M. Fleming, Nature (London) 392, 162 (1998).

${ }^{8}$ R. B. van Dover and L. F. Schneemeyer, Macromol. Rapid Commun. 25, 150 (2004).

${ }^{9}$ H. Chang, I. Takeuchi, and X. D. Xiang, Appl. Phys. Lett. 74, 1165 (1999).

${ }^{10}$ H. C. Theuerer and J. J. Hauser, J. Appl. Phys. 35, 554 (1964).

${ }^{11}$ R. B. van Dover, M. Hong, E. M. Gyorgy, J. F. Dillon, and S. D. Albiston, J. Appl. Phys. 57, 3897 (1985).

${ }^{12}$ E. Casado-Rivera, Z. Gal, A. C. D. Angelo, C. Lind, F. J. DiSalvo, and H. D. Abruña, Chem. Phys. Chem. 4, 193 (2003).

${ }^{13}$ E. Casado-Rivera et al., J. Am. Chem. Soc. 126, 4043 (2004).

${ }^{14}$ M. Prochaska, J. Jin, D. Rochefort, L. Zhuang, F. J. DiSalvo, H. D. Abruña, and R. B. van Dover, Rev. Sci. Instrum. 77, 054104 (2006).

${ }^{15}$ See EPAPS Document No. E-RSINAK-78-058707 for additional information on heater design. This document can be reached via a direct link in the online article's HTML reference section or via the EPAPS homepage (http://www.aip.org/pubservs/epaps.html).

${ }^{16}$ R. A. Waldo, M. C. Militello, and S. W. Gaarenstroom, Surf. Interface Anal. 20, 111 (2004).

${ }^{17}$ L. van der Pauw, Philips Res. Rep. 13, 1 (1958). 